\title{
Spatially Weighted Mutual Information (SWMI) for Registration of Digitally Reconstructed ex vivo Whole Mount Histology and in vivo prostate MRI
}

\author{
Pratik Patel, Jonathan Chappelow, John Tomaszewski, \\ Michael D. Feldman, Mark Rosen, Natalie Shih, Anant Madabhushi
}

\begin{abstract}
In this work, we present a scheme for the registration of digitally reconstructed whole mount histology (WMH) to pre-operative in vivo multiprotocol prostate MR imagery (T2w and DCE) using spatially weighted mutual information (SWMI). Spatial alignment of ex vivo histological sections to pre-operative in vivo MRI for prostate cancer $(\mathrm{CaP})$ patients undergoing radical prostatectomy is a necessary first step in the discovery of quantitative multiprotocol MRI signatures for CaP. This may be done by spatially mapping delineated extent of disease on ex vivo histopathology onto pre-operative in vivo MRI via image registration. Apart from the challenges in spatially registering multi-modal data (histology and MRI) on account of (a) modality specific differences, (b) deformation due to the endorectal coil and tissue loss on histology, another complication is that the ex vivo histological sections, in the lab, are usually obtained as quadrants. This means they need to be reconstituted as a pseudo-whole mount histologic section (WMHS) prior to registration with MRI. An additional challenge is that most registration techniques rely on availability of the pre-segmented prostate capsule on T2w MRI. The novel contribution of this paper is that it leverages a spatially weighted mutual information (SWMI) scheme to automatically register and map CaP extent from WMHS onto pre-operative, multiprotocol MRI. The SWMI scheme obviates the need for pre-segmentation of the prostate capsule on MRI. Additionally, we leverage a program developed by our group, Histostitcher c , for interactive stitching of individual histology quadrants to digitally reconstruct the pseudo WMHS. Our registration methodology comprises the following main steps, (1) affine registration of T2w and DCE MRI, (2) affine registration of stitched WMHS to multiprotocol T2w and DCE MRI, and (3) multimodal image registration of WMHS to multiprotocol T2w and DCE MRI using SWMI. We quantitatively and qualitatively evaluated all aspects of our methodology in the multimodal registration of a total of 7 corresponding histology and MRI sections from 2 different patients. For the 7 studies, we obtained an average Hausdorff distance of $1.85 \mathrm{~mm}$, mean absolute distance of 0.99 $\mathrm{mm}$, RMS of $1.65 \mathrm{~mm}$, and DICE of 0.83 , when comparing the capsular alignment on MRI to histology.
\end{abstract}

\section{INTRODUCTION}

Recently, magnetic resonance (MR) imaging (MRI) has emerged as a promising modality for detection of prostate

This work was made possible by grants on the Walter $\mathrm{H}$. Coulter Foundation, National Cancer Institute (Grant Nos. R01CA136535, R01CA140772, and R03CA143991), Department of Defense Prostate Cancer Research Program, The Cancer Institute of New Jersey and the Society for Imaging Informatics in Medicine.

Pratik Patel, Jonathan Chappelow, and Anant Madabhushi are with the Department of Biomedical Engineering at Rutgers, the State University of New Jersey, Piscataway, NJ 08854 anantmerci.rutgers.edu

John Tomaszewski, Michael D. Feldman, Mark Rosen, and Natalie Shih are with the Hospital of the University of Pennsylvania cancer $(\mathrm{CaP})$, with several studies showing that 3 Tesla (T) endorectal in vivo T2-weighted (T2-w) imaging yields significantly higher contrast and resolution compared to ultrasound (US) [1]. Quantitative integration of multiprotocol MRI (T2w, dynamic contrast enhanced (DCE), diffusion, spectroscopy) has been shown to result in significantly more accurate disease diagnosis compared to the individual protocols [2]. While the current clinical protocol for $\mathrm{CaP}$ screening does not involve in vivo diagnostic imaging, multiprotocol MRI would have significant implications for detection and localization of $\mathrm{CaP}$ which in turn could set the stage for (1) non-invasive image-based $\mathrm{CaP}$ screening, (2) targeted biopsies, and (3) conformal radiation therapy [2].

In order to identify quantitative multiprotocol MRI signatures for $\mathrm{CaP}$ extent, spatial extent of disease has to first be accurately ascertained on MRI [3]. The current "gold standard" for prostate cancer diagnosis is histology. Nearly 50,000 patients every year undergo radical prostatectomy after a positive diagnosis of $\mathrm{CaP}$. If a pre-operative MRI is performed in these patients prior to gland resection, then the in vivo imaging can be spatially aligned with the $e x$ vivo histologic specimen to map spatial extent of $\mathrm{CaP}$ onto corresponding multiprotocol MRI. This ground truth extent for disease on the MRI could then be used for quantitatively identify $\mathrm{CaP}$ specific multiprotocol MRI signatures.

Multimodal image registration of ex vivo $\mathrm{WMH}$ and in vivo MRI is complicated on account of a variety of image acquisition issues and due to tissue deformations. Ex vivo histology is deformed by the forces on the glass slide on which it is placed and by the internal forces of the sliced histology. In addition, the acquisition of the histological slices is prone to unequal slice to slice thickness and tissue loss. On the other hand, in vivo MRI is subject to deformations caused by the surrounding anatomy, such as the bladder in the anterior region and the endorectal coil in the posterior region. In addition, the digitization of histology requires the individual slices to be sectioned into quadrants. To the best of our knowledge, no scheme for direct multimodal registration of quadrants onto MRI is extant.

Most image registration methodologies are accomplished by three important criteria: 1) choosing a similarity measure, 2) choosing a transformation model, and 3) choosing an optimization strategy [4]. The goal of mutual information, a common choice of similarity measure, is to maximize the information shared between two images by minimizing the 


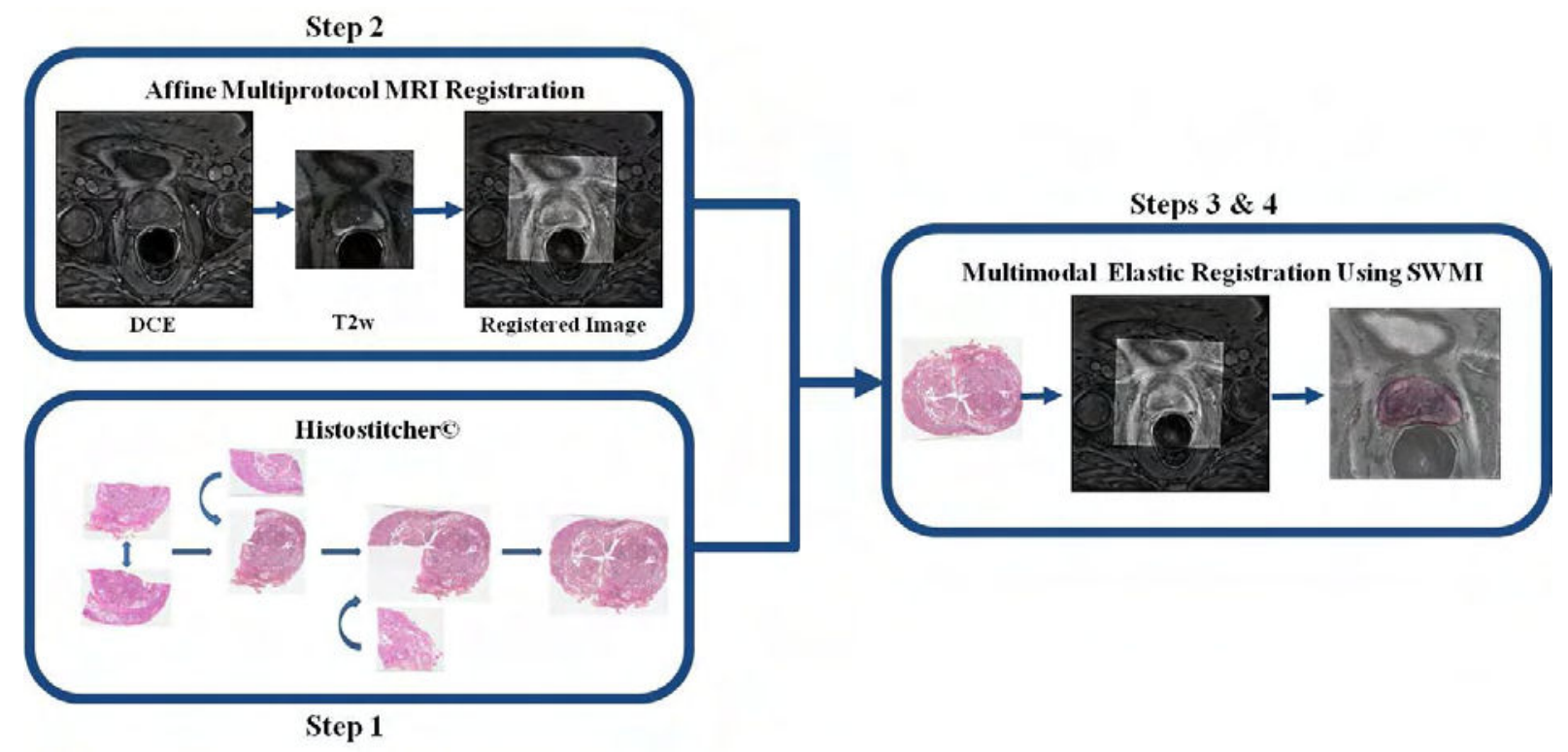

Fig. 1: Overview of the registration methodology for aligning ex vivo WMHS to pre-operative in vivo MRI. In Step 1, Histostitcher is used to stitch the individual histology quadrants. In Step 2, multiprotocol affine registration is performed using normalized mutual information (NMI). Step 3 involves the affine registration of histology with multiprotocol MRI. Finally, Step 4 deals with elastic registration employing SWMI without explicit segmentation of the prostate capsule on the in-vivo MRI.

joint entropy that is present between the two images [5]. While Park et al. [6] have developed methods to register ex vivo histology to multiprotocol MRI, the methods they employ are not practical for the clinical setting. Sophisticated methods for multimodal image registration have been developed to capture the deformations of histology onto MRI [7]; however, most of these state-of-the-art methods require the explicit segmentation of the prostate on MRI. Practically, acquiring segmentations is not desirable due to the large time commitment and inter-observer variability.

In order to derive a formulation which obviates the need for explicit segmentation of the prostate on MRI, it is necessary assign a certain part of the image as being higher in priority for alignment compared to other parts of the image. This can be achieved by spatially weighting the importance of registration in the calculation of MI. Previous attempts at weighting MI have been applied after the calculation of joint histogram space [8]. However, this formulation does not place spatial weighing on the calculation of MI because the joint histogram space provides statistical presence of pixel intensities and contains no information in regards to the spatial locations of these intensities. In order to incorporate spatial importance, weighting needs to be incorporated during the calculation of the joint histogram space [8].

Our novel contributions in this work are that we use spatially weighted mutual information (SWMI) to register and map disease extent and the prostate capsule from ex vivo histology to in vivo MRI. In doing so, we obviate the laborious task of explicit segmentation of prostate on MRI. In addition, we show how Histostitcher [9], developed by our group, can be leveraged to mosaic individual quadrants to form a pseudo WMH for multimodal image registration.

\section{Spatially Weighted Mutual Information}

For the remainder of the paper, let $\mathrm{T} 2 \mathrm{w}$ be represented by $\mathcal{A}$, histology by $\mathcal{B}$, and DCE by $\mathcal{D}$. In addition, we define all images as having integral values scaled from 0 to some pre-specified value.

\section{A. Mutual Information}

Conventional MI aims to find how closely two images are related using respective intensity values. The Shannon definition of entropy is commonly used to calculate the marginal and joint entropies between two images $\mathcal{A}$ and $\mathcal{B}$ [5]

$$
I(\mathcal{A}, \mathcal{B})=S(\mathcal{A})+S(\mathcal{B})-S(\mathcal{A}, \mathcal{B})
$$

where $S(\mathcal{A})$ and $S(\mathcal{B})$ are the respective marginal entropies. Furthermore, $S(\mathcal{A}, \mathcal{B})$ is the joint entropy of $S(\mathcal{A})$ and $S(\mathcal{B})$.

\section{B. Spatially Weighted Mutual Information}

In order to incorporate spatial weighting into the calculation of MI between $\mathcal{A}$ and $\mathcal{B}$, a weight matrix, $w$ is constructed and used for the calculation of the joint probability distribution. The weighting matrix is determined by assigning specific values for each pixel in accordance with the shape of the Gaussian function centered at the target region.

Weighting function $w(i) \in \mathbb{R}$ is defined for each pixel $i \in C$, where the number of pixels $N$ is defined as $N=|C|$. A joint density estimate for images $\mathcal{A}$ and $\mathcal{B}$ with pre-binned intensities (integral value quantization) at each pixel $\mathcal{A}(i) \in$ 
$\mathbb{N}$ and $\mathcal{B}(i) \in \mathbb{N}$, where $\mathbb{N}$ is the set of natural numbers, can be defined as,

$$
p_{\mathcal{A}, \mathcal{B}}(a, b)=\frac{1}{N} \sum_{i=1}^{N} w(i) \cdot I(\mathcal{A}(i)-a, \mathcal{B}(i)-b)
$$

where $a \in \mathbb{N}$ and $b \in \mathbb{N}$ span the range of integral valued intensities in $\mathcal{A}$ and $\mathcal{B}$. Additionally, $I$ is defined as the indicator function expressed as follows

$$
I(d a, d b)= \begin{cases}1, & \text { if } d a=0 \text { and } d b=0 \\ 0, & \text { otherwise }\end{cases}
$$

The shape of the Gaussian function is determined by selecting a rectangular region around the the target region centered at the prostate. The standard deviation of the Gaussian is dependent on the size of the rectangular region. The weighting function values a pixel inside the rectangular Gaussian region as 1 plus the value of the Gaussian at that pixel location. Meanwhile, pixels outside of the rectangular Gaussian region are valued at an importance of just 1 . After $p_{\mathcal{A}, \mathcal{B}}(a, b)$ is calculated, the Shannon joint entropy is calculated as follows:

$$
S(\mathcal{A}, \mathcal{B})=\sum_{a, b} p_{\mathcal{A}, \mathcal{B}}(a, b) \log p_{\mathcal{A}, \mathcal{B}}(a, b)
$$

The uniqueness of this formulation of the joint probability distribution is that the target region is given a higher spatial weighting when the joint probability distribution is calculated. Nonetheless, regions not considered to be within the target region are given equal weighting relative to each other, but overall lower compared to the target region.

\section{ExPerimental Design And Results}

\section{A. Brief Overview}

A brief overview of the methodology is as follows

Step 1. HistoStitcher is used to stitch high resolution histology sections to pseudo WMH,

Step 2. Multiprotocol Affine Registration is performed to bring DCE in alignment with T2w

Step 3. Multimodal Affine Registration is performed for an initial alignment of the pseudo WMH to MRI

Step 4. Multimodal Elastic Registration using SWMI is performed to capture non-rigid transformations between histology and MRI.

\section{B. Histostitcher}

Histostitcher was developed by Chappelow et al. [9] to interactively mosaic adjacent histological sections to construct a pseudo WMHS. The steps in using the interactive software are as follows:

Step 1. Load the stationary and moving histological section Step 2. Place control points at the boundaries of both the stationary and moving images corresponding to contiguous anatomy

Step 3. Preview transformation at low resolution for optimal alignment. If preview of alignment is not desired, remove control points and repeat Step 2 until optimal transformation in low resolution is found
Step 4. Confirm the low resolution alignment and press done to process the transformation at the higher resolution

The user can chose different levels of flexibility for the transformation of the moving image such as allowing for anisotropic scaling, reflection, rotation, and translation. Additionally, the user can restrict scaling and reflection and simply allow rotation and translation of the moving image with respect to the stationary image.

\section{Multiprotocol Affine Registration}

To bring $\mathcal{D}$ in alignment with $\mathcal{A}$, a 3D affine registration was carried out [7]. Nine parameters were optimized to ensure alignment of $\mathcal{D}$ with $\mathcal{A}$. Normalized mutual information, without the use of spatial weighting, was chosen as the similarity measure for optimization. The nine parameters that were optimized in the three planes were rotation, translation, and scaling. The highest weights were placed on rotation about the Z-axis and translation in $\mathrm{X}$-Y plane. To account for the different fields of view, $\mathcal{A}$ was padded to match the dimensions of $\mathcal{D}$.

\section{Multimodal Affine Registration}

Before elastic registration of $\mathcal{A}$ with $\mathcal{B}$ can be performed, it it crucial that ex vivo $\mathrm{WMH}$ is scaled and translated relatively to the size of the in vivo MRI. Histology was scaled down to the size of MRI using differences in areas of $\mathcal{A}$ and $\mathcal{B}$. Finally, to fine tune the non-elastic registration parameters, affine registration was performed by optimizing in plane scaling and rotation using normalized mutual information as the similarity measure [7].

\section{E. Multimodal Elastic Registration}

Affine registration is desirable for only an initial alignment of $\mathcal{A}$ to $\mathcal{B}$. Thus, elastic registration of stitched histological slices with $\mathrm{T} 2 \mathrm{w}$ was performed using free form deformations (FFDs) [7]. To ensure that large and small deformations were captured, FFDs using a a hierarchy of spline sizes were used to find find the optimal transformation. Using SWMI, the elastic registration procedure did not require the explicit segmentation of the prostate capsule on MRI.

\section{F. Results}

Figure 2 shows the registration result using SWMI. Table I shows the quantitative results of the SWMI driven registration by evaluating the boundary of the warped histology with the ground truth (manually segmented prostate capsule on MRI). The Hausdorff distance (HD) ranged from 1.23 $\mathrm{mm}$ to $2.4 \mathrm{~mm}$; mean absolute distance (MAD) ranged from $0.66 \mathrm{~mm}$ to $1.23 \mathrm{~mm}$ and the root mean squared distance (RMS) ranged from $1.05 \mathrm{~mm}$ to $2.03 \mathrm{~mm}$. In Figures 2(d) and 2(h), the warped pseudo histology appears to be well aligned with the prostate on MRI. The average of DICE's coefficient values is 0.83 , indicating a high percentage of overlap between the area of the deformed histology with the manual segmentation of prostate on MRI. The results presented demonstrate the ability of using SWMI to drive multimodal registration of pseudo WMHS to MRI. 


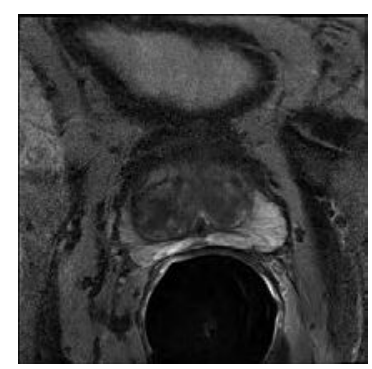

(a)

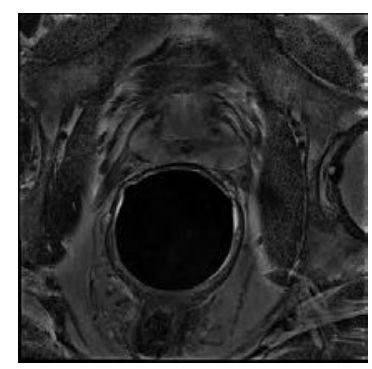

(e)
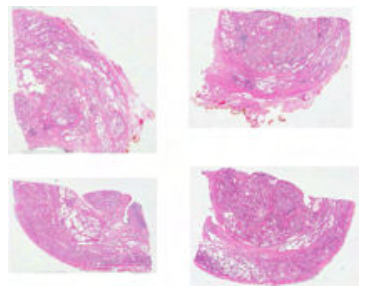

(b)

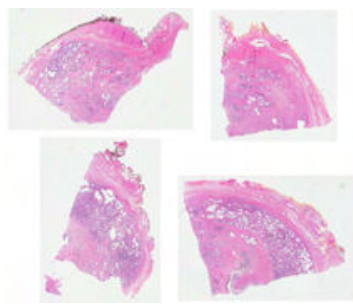

(f)

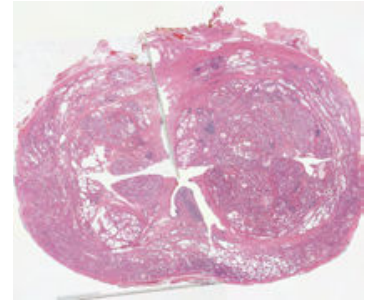

(c)

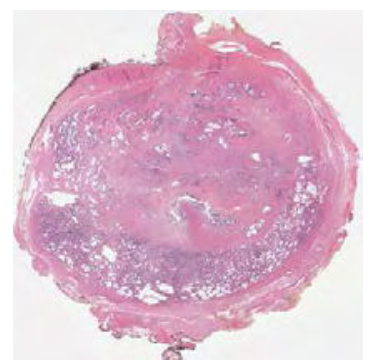

(g)

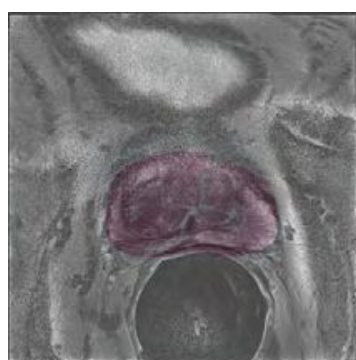

(d)

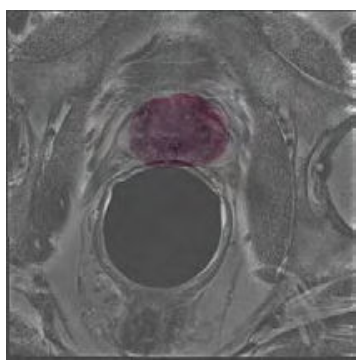

(h)

Fig. 2: Two studies for which histological fragments were stitched using Histostitcher and then registered to multiprotocol MRI are shown. (a)-(d) represent study 1 and (e)-(h) represent study 2. (a) and (e) are the initial in vivo MRI. (b) and (f) are histological fragments after digitization and are stitched to form pseudo WMH in (c) and (g). Finally, (d) and (h) show the final multimodal registration of the pseudo WMHs for study 1 and study 2, respectively.

\begin{tabular}{|c|c|c|c|c|}
\hline & $D_{H D}$ & $D_{M A D}$ & $D_{R M S}$ & $D_{\text {Dice }}$ \\
\hline Slice 1 & 1.23 & 1.23 & 2.33 & 0.89 \\
\hline Slice 2 & 2.23 & 1.19 & 2.20 & 0.81 \\
\hline Slice 3 & 2.40 & 1.06 & 1.68 & 0.86 \\
\hline Slice 4 & 2.03 & 0.98 & 1.53 & 0.81 \\
\hline Slice 5 & 2.26 & 1.01 & 1.53 & 0.69 \\
\hline Slice 6 & 1.46 & 0.80 & 1.24 & 0.89 \\
\hline Slice 7 & 1.35 & 0.66 & 1.05 & 0.88 \\
\hline Average Values & 1.85 & 0.99 & 1.65 & 0.83 \\
\hline
\end{tabular}

TABLE I: Quantitative evaluation of SWMI driven registration for 7 multimodal histologic, multi protocol MRI studies from 2 patients. Individual evaluations and average values of $\mathrm{HD}, \mathrm{MAD}$, and RMS are provided in millimeters.

The total time required for WMHS stitching and multimodal elastic registration varies from slice to slice. The largest amount of time is required for the stitching of the WMHS.

\section{CONCLuding Remarks}

We have presented a novel application of spatially weighted mutual information for registration of multimodal image registration of pseudo whole mount histological sections to multiprotocol MRI. The digital reconstitution of pseudo WMH using Histostitcher from histological sections enabled accurate elastic registration of WMH to multiprotocol MRI. Using SWMI, multimodal image registration of pseudo WMH with multiprotocol MRI without explicit prostate capsule segmentation on MRI was demonstrated. In addition, using SWMI to register histology with multiprotocol MRI was found to be highly accurate in terms of the Hausdorff distance, mean absolute distance, root mean squared distance, and DICE. Future work will involve using results from SWMI driven multimodal image registration to automatically initiate iterative segmentation-registration schemes [10].

\section{REFERENCES}

[1] B. Bloch et al., "Prostate cancer : Accurate determination of extracapsular extension with high-spatial-resolution dynamic contrastenhanced and $\mathrm{t} 2$-weighted $\mathrm{mr}$ imaging initial results," Radiology, vol. 245, pp. 176-185, 2007.

[2] G. Xiao et al., "Determining histology-mri slice correspondences for defining mri-based disease signatures of prostate cancer." ComputMedImagingGraph, 2011.

[3] S. Viswanath et al., "A comprehensive segmentation, registration, and cancer detection scheme on 3 tesla prostate dce mri," in MICCAI, 2008, vol. 5241, pp. 662-669.

[4] F. Maes, A. Collignon et al., "Multimodality image registration by maximization of mutual information," Medical Imaging, IEEE Transactions on, vol. 16, no. 2, pp. 187 -198, 1997.

[5] J. Pluim et al., "Mutual-information-based registration of medical images: a survey," Medical Imaging, IEEE Transactions on, vol. 22, no. 8, pp. $986-1004,2003$.

[6] H. Park et al., "Registration methods for histological slides and ex vivo mri of prostate," in Nuclear Science Symposium Conference Record, 2007. NSS '07. IEEE, vol. 4, 26 2007-nov. 3 2007, pp. 3102 -3104.

[7] J. Chappelow et al., "Elastic registration of multimodal prostate mri and histology via multiattribute combined mutual information," Medical Physics, vol. 38, pp. 2005-2018, 2011.

[8] S. B. Park et al., "Spatially weighted mutual information image registration for image guided radiation therapy," Medical Physics, vol. 37, no. 9, pp. 4590-4601, 2010.

[9] J. Chappelow, A. Madabhushi et al., "Histostitcher: An interactive program for accurate and rapid reconstruction of digitized whole histological sections from tissue fragments," Computerized Medical Imaging and Graphics, vol. In Press, Corrected Proof, 2011.

[10] K. K. Bhatia et al., "Groupwise combined segmentation and registration for atlas construction." Med Image Comput Comput Assist Interv, vol. 10, no. Pt 1, pp. 532-540, 2007. 